\title{
Vi raccontiamo la qualunque
}

\author{
Claudio Giovanardi
}

PUBBLICATO: 06 MARZO 2020

\section{Quesito:}

Alcuni lettori ci chiedono "delucidazioni" in merito all'uso dell'espressione la qualunque per intendere 'qualunque cosa', 'qualunque persona': è corretto? Da dove deriva?

\section{Vi raccontiamo la qualunque}

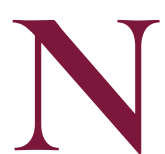

on vi è alcun dubbio che l'espressione la qualunque per intendere 'qualunque cosa' sia stata favorita dal grande successo di un personaggio televisivo e cinematografico interpretato dal comico lombardo Antonio Albanese, alias Cetto La Qualunque, maschera che rappresenta un politico calabrese avido e corrotto. Lintenzione satirica, anche a livello linguistico, è del resto evidente già nel titolo del film del zor che lanciò sul grande schermo il personaggio in questione, ovvero Qualunquemente (regia di Giulio Manfredonia), ed è ribadita nel recentissimo film intitolato Cetto c'é, senzadubbiamente (2019, regia ancora di Giulio Manfredonia). Sarà bene, dunque, sottolineare in partenza che nelle intenzioni di chi usa la qualunque in un contesto discorsivo "normale" vi è (almeno sino ad oggi) il voler evidenziarne la carica espressiva pur nella consapevolezza che si tratta di un uso scorretto. Chi dice (o, meno comunemente, scrive) la qualunque, è ben conscio di commettere una forzatura grammaticale, che però gli torna utile per rafforzare o dotare di maggiore espressività un concetto o un argomento.

Recentemente Vittorio Coletti ha richiamato l'attenzione degli studiosi sulla difficoltà di riunire sotto la medesima etichetta grammaticale i cosiddetti "indefiniti", una categoria eterogenea e difficilmente riconducibile a sintesi, il cui elemento comune pare essere la quantificazione (cfr. Coletti 2018). A proposito di qualunque e degli altri "indeterminativi di qualità", lo studioso afferma che in essi "la vaghezza semantica è un tratto essenziale del giudizio (specie svalutativo o di indifferenza) verso il nome cui si agganciano o che sostituiscono" (p. 196). Emerge dunque con chiarezza che qualunque, qualsiasi, chiunque ecc., oltre al valore semantico possiedono spesso un sovrappiù di effetto pragmatico rispetto al nome di riferimento. Cio si rileva facilmente considerando che la posizione dell'aggettivo indeterminativo di qualità rispetto al nome è determinante per la selezione di diversi significati; se, infatti, una qualunque persona indica una persona non definita, una persona qualunque allude piuttosto alle caratteristiche di mediocrità di un individuo in particolare. Detto in altri termini: "quando qualunque e qualsiasi sono accompagnati dall'articolo indeterminativo e sono posposti al nome perdono il valore di determinanti indefiniti e assumono quello di aggettivi qualificativi, con il significato di 'ordinario"' (cfr. Cordin 2019, p. 86). Patrizia Cordin, dal canto suo, sussume gli indefiniti nella categoria più generale dei quantificatori e definisce la classe cui appartiene qualunque "indefiniti distributivi", laddove Prandi aveva preferito "indefiniti di qualità" (cfr. rispettivamente Cordin 2019, p. 77 e Prandi 2006, p. 298).

Tornando a la qualunque, il rapido excursus grammaticale consente di rispondere ai quesiti posti. Sulla mancata liceità in contesti formali non ci sono dubbi. Resta tuttavia inteso che la qualunque è sempre usato con sottolineatura ironica (e spesso spregiativa) e consapevolezza d'infrazione della norma grammaticale vigente; ciò è ulteriormente dimostrato dal fatto che non si tratta (almeno sino ad ora) 
di un uso popolare, ma di un vezzo delle persone mediamente istruite o addirittura colte. Va aggiunto che, secondo reperti ancor più recenti, oltre a la qualunque si sta diffondendo anche la formula la qualsiasi. Non pare che l'uso sia limitato a una determinata area e che quindi risuoni solo in alcuni italiani regionali, ma che piuttosto si tratti di un tipo panitaliano. Quanto infine all'origine, è forse ipotizzabile che, a partire dall'espressione corretta una qualunque $\cos a$, si sia avuta una sostituzione dell'articolo indeterminativo una con il corrispettivo determinativo la (*la qualunque cosa), e che poi, per ellissi del sostantivo, si sia arrivati a la qualunque.

Nota bibliografica:

- Coletti 20r8: Vittorio Coletti, L'inafferrabile indeterminatezza degli indefiniti, in "Acció che 'l nostro dire sia ben chiaro". Scritti per Nicoletta Maraschio, a cura di Marco Biffi, Francesca Cialdini, Raffaella Setti, vol. I, Firenze, Accademia della Crusca, 20I8, pp. I95-203.

- Cordin 2019: Patrizia Cordin, Pronomi e determinanti, Roma, Carocci, 2019.

- Prandi 2006: Michele Prandi, Le regole e le scelte. Introduzione alla grammatica italiana, Torino, Utet, 2006

\section{Cita come:}

Claudio Giovanardi, Vi raccontiamo la qualunque , "Italiano digitale", 2019, XII, 2020/1 (gennaiomarzo)

DOI: $10.35948 / 2532-9006 / 2020.3262$

Copyright 2019 Accademia della Crusca

Pubblicato con licenza creative commons CC BY-NC-ND 